\title{
Wertpapierbörsen im Internet - Informationsangebot aus Sicht des Privatanlegers
}

Peter Gomber, Carsten Holtmann, Christine Kurbjuweit, Silja Moderer ${ }^{1}$

\section{Stichworte:}

Börsen, Wertpapierhandel, Kursdaten, real-time Kurse, Privatanleger

\section{Zusammenfassung:}

Dieser Beitrag gibt einen Überblick zum Informationsangebot der wichtigsten Wertpapierbörsen aus Sicht des deutschen Privatanlegers. Untersucht werden sowohl internationale Märkte als auch die deutschen Regionalbörsen. Es zeigt sich, daß die Marktbetreiber sich sowohl bezüglich des Informationsangebotes als auch bezüglich der Informationsaufbereitung deutlich unterscheiden. Die Informationsbedarfe institutioneller Anleger stehen - trotz des verbesserten Angebotes für Privatinvestoren - bei vielen Institutionen im Vordergrund ihres Webauftrittes.

\section{Stock Exchanges on the Internet - Information supplied from the private investor's point of view}

\section{key words:}

stock exchanges, securities trading, transaction data, real-time data, private investor

\section{abstract:}

This paper gives an overview of the information supplied to private investors by the most important international as well as German regional stock exchanges. Results of the analysis show that the various stock exchanges differ significantly concerning information supply and processing . Despite the fact that stock exchanges have improved the information supply for

\footnotetext{
${ }^{1}$ Dr. Peter Gomber, Dipl. Kfm. Carsten Holtmann, Lehrstuhl für BWL-Wirtschaftsinformatik, Universität Gießen, Licher Str. 70, D-35394 Gießen, E-Mail: \{peter.gomber|carsten.v.holtmann\}@wirtschaft.uni-giessen.de Christine Kurbjuweit, Auf dem Erbel 16, 35764 Sinn, E-Mail: Christine.Kurbjuweit@gmx.de Silja Moderer, Ockershäuser Allee 48, 35039 Marburg, E-Mail: silja_moderer@hotmail.com
} 
private investors, many institutions continue to focus mainly on the specific needs of institutional investors.

\section{Einleitung}

Die Globalisierung der Kapitalströme und die rasante Entwicklung auf dem Gebiet der Informations- und Kommunikationstechnologie verschärfen bereits seit einigen Jahren den Wettbewerb der Börsenplätze auf nationaler und internationaler Ebene [Birk96; Metz94].

Das Internet spielt in diesem Zusammenhang eine besondere Rolle: Einerseits entwickeln sich auf dessen technologischer Basis in zunehmendem Maße Alternativen zum börslichen Wertpapierhandel in Form privater elektronischer Handelssysteme, die auch als Electronic Communication Networks oder Proprietary Trading Systems bezeichnet werden, und deren Existenz den Wettbewerbsdruck für die etablierten Börseninstitutionen weiter erhöht [voRo94; Go2000]. Andererseits - und das soll Inhalt der folgenden Betrachtungen sein bietet das Internet über die konventionellen Kommunikationswege hinaus einen weiteren Vertriebsweg. Dabei wird es von den renommierten Börsen primär zur Informationsbereitstellung verwendet; die Durchführung von Transaktionen wird im börslichen Handel bisher nur über Zugangsintermediäre (z. B. Online-Broker) angeboten [WeGo99].

Über das Internet ist es den Börsen möglich, mit seriösen, relevanten und aktuellen Informationen $\mathrm{zu}$ den von ihnen angebotenen Produkten und Services das Interesse und Vertrauen von (Privat-) Investoren zu erlangen bzw. das bereits aufgebaute Reputationskapital als „trusted parties“ bei der Informationsbereitstellung aufrechtzuerhalten, um auch in Zukunft Orders auf ihre Handelsplattformen zu ziehen [WeGo99].

Die Kundengruppe privater Investoren hat in den letzten Jahren stark an Bedeutung gewonnen und zeichnet sich durch eine zunehmende Nachfrage nach Informationen als Entscheidungshilfe für den Wertpapierhandel aus. Höhere Vermögen und eine verbesserte Kenntnis finanzmarkt-theoretischer Zusammenhänge führen $\mathrm{zu}$ einem deutlichen Anstieg privater Wertpapierinvestitionen und einer Umgehung der traditionellen Intermediäre durch die Nutzung von Online-Brokern.

Vor diesem Hintergrund werden im Folgenden die Internetangebote zehn internationaler Börseninstitutionen sowie der deutschen Regionalbörsen näher beleuchtet, um zu vergleichen, 
- welche Informationen von den einzelnen Börseninstitutionen auf ihren Internetsites bereitgestellt werden und

- in welchem qualitativen und quantitativen Umfang dies geschieht.

In Kapitel 2 wird dazu auf die Vorgehensweise bei der Auswahl der betrachteten Börseninstitutionen und die gewählten Beurteilungskriterien eingegangen, bevor im Kapitel 3 der konkrete Vergleich stattfindet. Kapitel 4 faßt die Aussagen abschließend zusammen.

Die folgenden Ausführungen beziehen sich ausschließlich auf Kassamärkte, da diese für den privaten Investor primär von Interesse sind.

\section{Vorgehensweise}

Im folgenden Abschnitt 2.1 wird die Auswahl der zu vergleichenden Börsen begründet. Abschnitt 2.2 stellt dann die Beurteilungskriterien dar, nach denen die einzelnen Börsenwebsites untersucht werden.

\subsection{Auswahl der Börseninstitutionen}

Die folgende Tabelle 1 zeigt zehn aus Sicht des Privatanlegers relevante internationale Börseninstitutionen mit Angabe ihres Sitzes sowie die sieben deutschen Regionalbörsen mit Angabe der Umsätze ${ }^{2}$ und der jeweiligen URLs. Die folgenden Ausführungen basieren auf Erhebungen im Dezember 1999.

\begin{tabular}{|l|l|l|l|}
\hline \multicolumn{1}{|c|}{ NAME } & \multicolumn{1}{|c|}{ LAND } & \multicolumn{1}{|c|}{$\begin{array}{c}\text { UMSÄTZE IN } \\
\text { AKTIEN 1998 IN } \\
\text { MIO. US-\$ [DAI99] }\end{array}$} & \multicolumn{1}{c|}{$\begin{array}{c}\text { URL } \\
\text { http://www. }\end{array}$} \\
\hline Amsterdam Exchange & NL & $405.217,3$ & aex.nl \\
\hline Bolsa de Madrid & E & $640.320,3$ & bolsamadrid.es \\
\hline Borsa Italia ${ }^{3}$ & I & $486.506,7$ & borsaitalia.it \\
\hline Bourse de Paris & F & $2.053 .299,8$ & bourse-de-paris.fr \\
\hline $\begin{array}{l}\text { Frankfurter } \\
\text { Wertpapierbörse } \\
\text { (FWB) }\end{array}$ & D & $1.491 .795,7$ & exchange.de \\
\hline $\begin{array}{l}\text { London Stock } \\
\text { Exchange }\end{array}$ & GB & $2.887 .989,9$ & londonstockex.co.uk \\
\hline $\begin{array}{l}\text { National Association } \\
\text { of Security Dealers }\end{array}$ & USA & $5.518 .946,3$ & nasdaq.com \\
\hline
\end{tabular}

2 Eine direkte Vergleichbarkeit der Umsätze zwischen den aufgeführten Börsen ist aufgrund unterschiedlicher Ermittlungsverfahren nicht möglich [DAI99].

${ }^{3}$ Alle Ergebnisse der Analyse bezüglich der Borsa Italia beziehen sich auf den Stand Dezember 1998, da die Website zum Zeitpunkt der aktuellen Analyse im Dezember 1999 neu strukturiert und gestaltet wurde und ausschließlich in italienischer Sprache verfügbar war. 


\begin{tabular}{|c|c|c|c|}
\hline $\begin{array}{l}\begin{array}{l}\text { Automated Quotation } \\
\text { (NASDAQ) }\end{array} \\
\end{array}$ & & & \\
\hline $\begin{array}{l}\text { New York Stock } \\
\text { Exchange (NYSE) }\end{array}$ & USA & $7.317 .948,5$ & nyse.com \\
\hline Swiss Exchange & $\mathrm{CH}$ & $689.169,9$ & swX.com \\
\hline $\begin{array}{l}\text { Tokyo Stock } \\
\text { Exchange }\end{array}$ & $\mathrm{J}$ & 750.825 .2 & tse.or.jp \\
\hline NAME & LAND & $\begin{array}{c}\text { UMSÄTZE IN } \\
\text { AKTIEN } 1999 \text { IN } \\
\text { MIO. DM [DB2000] }\end{array}$ & $\begin{array}{c}\text { URL } \\
\text { http://www. }\end{array}$ \\
\hline $\begin{array}{l}\text { Baden- } \\
\text { Württembergische } \\
\text { Börse zu Stuttgart }\end{array}$ & $\mathrm{D}$ & $109.591,3$ & boerse-stuttgart.de \\
\hline Bayerische Börse & $\mathrm{D}$ & $74.570,6$ & bayerischeboerse.de \\
\hline Berliner Börse & $\mathrm{D}$ & $96.105,2$ & berlinerboerse.de \\
\hline $\begin{array}{l}\text { Bremer } \\
\text { Wertpapierbörse }\end{array}$ & $\mathrm{D}$ & $10.111,7$ & boerse-bremen.de \\
\hline $\begin{array}{l}\text { Hanseatische } \\
\text { Wertpapierbörse } \\
\text { Hamburg }\end{array}$ & $\mathrm{D}$ & $34.764,0$ & boerse-hamburg.de \\
\hline $\begin{array}{l}\text { Niedersächsiche Börse } \\
\text { zu Hannover }\end{array}$ & $\mathrm{D}$ & $4.294,3$ & boerse-hannover.de \\
\hline $\begin{array}{l}\text { Rheinisch- } \\
\text { Westfälische Börse zu } \\
\text { Düsseldorf }\end{array}$ & $\mathrm{D}$ & $67.499,9$ & rwb.de \\
\hline
\end{tabular}

Tabelle 1: Untersuchte Börseninstitutionen

\subsection{Erläuterung der Bewertungskriterien}

Zur Beurteilung der Qualität des Webauftrittes der Börseninstitutionen soll nicht die generelle Eignung des Internets im Vergleich zu anderen Medien [GrBi98], sondern die Vor- und Nachteile der einzelnen Auftritte aus Sicht eines privaten Investors vergleichend dargestellt werden. Dazu werden die im folgenden erläuterten Kriterien herangezogen:

\section{Funktionalität}

In dieser Kategorie werden alle Aspekte untersucht, die dem privaten Anleger eine möglichst komfortable und einfache Nutzung, d. h. einfache Navigation und leichtes Verständnis der Inhalte der Websites ermöglichen. Relevant sind im weiteren

- die Existenz von Sitemaps,

- das Vorhandensein eines Glossars für börsenspezifische Begriffe,

- die verfügbare(n) Sprache(n),

- die Existenz von Suchmaschinen für die zielgerichtete Suche von Informationen oder Kursen sowie

das Angebot spezieller Informationen mit Passwortschutz. 


\section{Informationsspektrum}

Während in der vorangegangenen Kategorie analysiert wird, wie der Zugang zu den Informationen realisiert ist, geht es hierbei darum, welche Informationen von der jeweiligen Börse in welcher Tiefe im Internet angeboten werden. Das Spektrum reicht von Kursdaten einzelner Wertpapiere und Indizes bis hin zu spezifischen Hintergrundinformationen zu den einzelnen notierten Unternehmen sowie den Börseninstitutionen selbst.

\section{Zeitnähe}

Mittels dieses Kriteriums wird geprüft, ob und mit welcher zeitlichen Verzögerung die Börsen Echtzeitkurse bzw. -indizes ${ }^{4}$ für den privaten Investor anbieten. Dieses Kriterium wurde ergänzend $\mathrm{zu}$ dem des Informationsspektrums gewählt, da es - aufgrund der vorne angesprochenen Entwicklungen - auch für den Privatinvestor zunehmend wichtig ist, zeitnah über Kursveränderungen und deren Hintergründe informiert $\mathrm{zu}$ sein, um schnell entsprechende Kauf- bzw. Verkaufsentscheidungen treffen zu können

\section{Vergleich}

\subsection{Funktionalität}

Die folgenden Ausführungen, Tabelle 2 sowie Bild 1 stellen die Resultate des Vergleiches im Hinblick auf die Funktionalität der jeweiligen Websites dar.

Bei ca. $70 \%$ aller untersuchten Börsen ist eine Sitemap vorhanden, die dem Besucher der Website die Navigation erleichtern soll. Allerdings besteht für den privaten Investor das Problem, daß in diesen Sitemaps oft nicht genau ersichtlich ist, was sich hinter einzelnen börsen- bzw. institutionsspezifischen Ausdrücken oder Abkürzungen verbirgt, da nur ca. 35\% über ein Glossar verfügen, das bestimmte Termini und Abkürzungen erläutert. Allerdings bieten die Bourse de Paris wie auch die Amsterdam Exchange ihre Glossare nur auf französisch bzw. niederländisch an, was sich für den privaten Investor dann als Nachteil darstellt, wenn dieser Erklärungsbedarf hat, ohne der Landessprache mächtig zu sein.

Bezüglich der Sprache ist weiterhin anzumerken, daß außer fünf Regionalbörsen alle Institutionen ihre Websites zusätzlich auf Englisch anbieten, falls dies nicht auch die Landessprache ist. Diesbezüglich sind bei der Amsterdam Exchange Defizite zu beobachten,

\footnotetext{
${ }^{4}$ Aufgrund der großen Anzahl möglicher Indizes beschränken wir uns aus Übersichtlichkeitsgründen auf Aktienindizes.
} 
da Informationen auf Englisch angekündigt werden, die entsprechenden Texte jedoch nur in holländischer Sprache erhältlich sind.

Die größten internationalen Börsen sind generell mit einer Suchmaschine für Stichwörter ausgestattet, die ein komfortables Auffinden der gewünschten Informationen ermöglichen.

Hinsichtlich der zielgerichteten Kurssuche fallen die Ergebnisse weit auseinander. Während die gezielte Suche nach einzelnen Indizes und bestimmten Kursen bei einigen Börsen grundsätzlich nicht möglich ist, ist diese bei anderen mittels spezieller Suchmaschinen sehr einfach und komfortabel. Zur letzten Kategorie gehören eindeutig die Websites der NYSE, NASDAQ, FWB und der Bourse de Paris.

An der NYSE und der Bourse de Paris können sich Privatinvestoren kostenlos registrieren, um sich nach Eingabe eines Passwortes ein individuelles Portefeuille zusammenzustellen und dessen Entwicklung zu beobachten. Bei der FWB können nach Passworteingabe Marktstatistiken abgerufen werden.

\begin{tabular}{|c|c|c|c|c|c|c|}
\hline NAME & SITEMAP & GLOSSAR & SPRACHE & $\begin{array}{l}\text { SUCHMASCHINE } \\
\text { FÜR BEGRIFFE }\end{array}$ & $\begin{array}{c}\text { SUCHMASCHINE } \\
\text { FÜR KURSE }\end{array}$ & PASSWORT \\
\hline $\begin{array}{l}\text { Amsterdam } \\
\text { Exchange }\end{array}$ & • & $\bullet^{1}$ & Engl./Holl. & & • & • \\
\hline Bolsa de Madrid & & & Engl./Span. & & & \\
\hline Borsa Italia & & & Engl./Ital. & $\bullet$ & $\bullet$ & \\
\hline Bourse de Paris & $\bullet$ & $\bullet^{2}$ & Engl./Frz. & $\bullet$ & $\bullet$ & $\bullet$ \\
\hline $\begin{array}{l}\text { Frankfurter } \\
\text { Wertpapierbörse }\end{array}$ & 3 & & Engl./Dt. & $\bullet$ & $\bullet$ & • \\
\hline $\begin{array}{l}\text { London Stock } \\
\text { Exchange }\end{array}$ & - & - & Engl. & 3 & & \\
\hline NASDAQ & $\bullet$ & $\bullet$ & Engl. & $\bullet$ & $\bullet$ & \\
\hline NYSE & $\bullet$ & $\bullet$ & Engl. & $\bullet$ & $\bullet$ & $\bullet$ \\
\hline Swiss Exchange & $\bullet$ & & Engl./Dt./Frz. & & & \\
\hline $\begin{array}{l}\text { Tokyo Stock } \\
\text { Exchange } \\
\end{array}$ & - & & Engl./Japan. & - & - & \\
\hline $\begin{array}{l}\text { Baden- } \\
\text { Württembergische } \\
\text { Börse zu Stuttgart } \\
\end{array}$ & $\bullet$ & • & Dt. & • & $\bullet$ & \\
\hline Bayerische Börse & $\bullet$ & & Engl./Dt. & & $\bullet$ & \\
\hline Berliner Börse & $\bullet$ & & Engl./Dt. & & $\bullet$ & \\
\hline $\begin{array}{l}\text { Bremer } \\
\text { Wertpapierbörse } \\
\end{array}$ & & & Dt. & & • & \\
\hline $\begin{array}{l}\text { Hanseatische } \\
\text { Wertpapierbörse } \\
\text { Hamburg }\end{array}$ & $\bullet$ & & Dt. & & • & - \\
\hline $\begin{array}{l}\text { Niedersächsiche } \\
\text { Börse zu Hannover }\end{array}$ & $\bullet$ & & Dt. & & & \\
\hline $\begin{array}{l}\text { Rheinisch- } \\
\text { Westfälische Börse } \\
\text { zu Düsseldorf }\end{array}$ & & & Dt. & & $\bullet$ & \\
\hline
\end{tabular}


${ }^{1}$ nur auf niederländisch erhältlich ${ }^{2}$ nur auf französisch erhältlich ${ }^{3} \mathrm{im}$ Aufbau

Tabelle 2: Funktionalität der Websites

\subsection{Informationsspektrum}

In Tabelle 3 sind die Resultate der Untersuchung bezüglich des Informationsspektrums der jeweiligen Börsen dargestellt. Im Folgenden werden generelle Tendenzen und Beispiele, die im positiven oder auch im negativen Sinne herausstechen, dargestellt.

Die Kursdaten einzelner Wertpapiere oder Indizes werden mit Ausnahme der London Stock Exchange - dort können diese nur über den „Umweg“ über andere Site-Anbieter (z.B. die BBC-Website: http://news.bbc.co.uk/hi/english/business/default.htm) abgerufen werden - von allen Institutionen zur Verfügung gestellt. Historische Kurse dagegen werden von nur ca. zwei Drittel der Börsenplätze angeboten, wobei die jeweiligen Zeiträume der Bereitstellung historischer Kursdaten teilweise erheblich differieren. Die jeweiligen Börsen bieten generell die wichtigsten börseneigenen bzw. nationalen Indizes an. Neben diesen stellen ca. 40\% zusätzlich die wichtigsten internationalen Indizes wie Dow Jones, DAX etc. bereit.

Im Hinblick auf Marktanalysen und -statistiken sowie Informationen zu den notierten Unternehmen wird deutlich, daß das Informationsspektrum je nach Börse und ihrer Größe sehr unterschiedlich ist: Tendenziell bieten die großen Institutionen dem Investor ein breites Spektrum an Marktanalysen sowie Statistiken und Daten zu den notierten Unternehmen. Diese enthalten außer dem Corporate Profile u.a. auch Bilanzdaten und Branchenstatistiken. Gute Beispiele hierfür sind die Websites der Bourse de Paris sowie von NASDAQ, NYSE und FWB, die sowohl qualitativ als auch quantitativ wertvolle Informationen für private Investoren bereitstellen. Negativ fiel der Börsenplatz London auf, da hier nur generelle Informationen über die Organisation der London Stock Exchange und ihrer Märkte angeboten werden.

Bezüglich zusätzlicher Services für Privatinvestoren sind Börseninstitutionen wie NYSE, Tokyo Stock Exchange und die Bourse de Paris positiv zu beurteilen, die sehr umfangreiche und ausführliche Daten in individuellen Gestaltungsformen anbieten. Weitere Dienste wie Marktsimulationen, Investor-Training oder Seminare, die für potentielle Anleger interessant sein können, werden von NASDAQ, der Stuttgarter und der Hamburger Börse bereitgestellt.

\begin{tabular}{|c|c|c|c|c|c|}
\hline NAME & KURS- & HISTO- & INDIZES & MARKTANALYSEN \\
DATEN & $\begin{array}{c}\text { RISCHE } \\
\text { KURS- } \\
\text { DATEN }\end{array}$ & & $\begin{array}{c}\text { ZUSÄTZLICHE } \\
\text { SERVICES FÜR } \\
\text { SOWIE } \\
\text { INFORMATIONEN } \\
\text { ZU DEN } \\
\text { NOTIERTEN }\end{array}$ & $\begin{array}{c}\text { PRIVATINVESTOREN } \\
\end{array}$ \\
& & & & \\
\hline
\end{tabular}




\begin{tabular}{|c|c|c|c|c|c|}
\hline & & & & UNTERNEHMEN & \\
\hline $\begin{array}{l}\text { Amsterdam } \\
\text { Exchange }\end{array}$ & $\bullet$ & $\bullet$ & $\begin{array}{l}\text { Nationale (AEX, } \\
\text { AMX, etc.) und } \\
\text { wichtige } \\
\text { internationale Indizes } \\
\text { (Dow Jones, DAX, } \\
\text { FTSE, etc.) }\end{array}$ & $\begin{array}{l}\text { Hitlisten, Statistiken, } \\
\text { Emittenten-Porträt, } \\
\text { Company News } \\
\text { (Bilanzprofil) }\end{array}$ & \\
\hline Bolsa de Madrid & $\bullet$ & 1 & $\begin{array}{l}\text { Nationale (IBEX } 35 \text {, } \\
\text { IGBM, etc.) und } \\
\text { internationale Indizes } \\
\text { (Dow Jones, etc.) }\end{array}$ & $\begin{array}{l}\text { Relevante } \\
\text { Marktanalysen und - } \\
\text { statistiken } \\
\text { kostenpflichtig bzw. } \\
\text { über andere Medien } \\
\text { erhältlich, Emittenten- } \\
\text { Porträts }\end{array}$ & $\begin{array}{l}\text { WWW-Links zu Maklern } \\
\text { und Finanzinstitutionen, } \\
\text { Fact Book }\end{array}$ \\
\hline Borsa Italia & $\bullet$ & $\bullet$ & $\begin{array}{l}\text { Nationale Indizes } \\
\text { (MIBTEL, MIB 30, } \\
\text { etc.) }\end{array}$ & $\begin{array}{l}\text { Marktstatistiken zu } \\
\text { Indizes und gehandelten } \\
\text { Produkten, } \\
\text { Marktindikatoren, } \\
\text { Hitliste, } \\
\text { Marktnachrichten, } \\
\text { Daten zu notierten } \\
\text { Unternehmen } \\
\end{array}$ & \\
\hline Bourse de Paris & $\bullet$ & $\bullet$ & $\begin{array}{l}\text { Nationale (CAC 40, } \\
\text { SBF 100, etc.) und } \\
\text { wichtige } \\
\text { internationale Indizes } \\
\text { (NASDAQ } \\
\text { Composite, FTSE, } \\
\text { Nikkei, etc.) }\end{array}$ & $\begin{array}{l}\text { Hitlisten, Daten zu } \\
\text { notierten Unternehmen, } \\
\text { Branchenstatistiken, ad- } \\
\text { hoc Meldungen }\end{array}$ & Pressedossier \\
\hline $\begin{array}{l}\text { Frankfurter } \\
\text { Wertpapierbörse }\end{array}$ & $\bullet$ & $\bullet$ & $\begin{array}{l}\text { Nationale Indizes } \\
\text { (DAX, DAX 100, } \\
\text { etc.), EURO.NM, } \\
\text { Dow Jones STOXX }\end{array}$ & $\begin{array}{l}\text { Ad-hoc Meldungen, } \\
\text { Marktstatistiken, } \\
\text { Hitlisten, Emittenten- } \\
\text { Kurzporträts und } \\
\text { Stammdaten }\end{array}$ & $\begin{array}{l}\text { Factbook, } \\
\text { Pressemitteilungen, } \\
\text { Newsletter, 1x1 der Börse, } \\
\text { Online-Magazin, } \\
\text { Newsboard, } \\
\text { Veranstaltungskalender, } \\
\text { WWW-Links zu } \\
\text { Finanzinstitutionen und } \\
\text { internationalen Börsen } \\
\end{array}$ \\
\hline $\begin{array}{l}\text { London Stock } \\
\text { Exchange }\end{array}$ & & & & Marktstatistiken & $\begin{array}{l}\text { Pressemitteilungen, } \\
\text { Publikationsübersicht, } \\
\text { WWW-Links zu } \\
\text { Finanzinstitutionen, } \\
\text { Informationen zu } \\
\text { Neuentwicklungen (SETS, } \\
\text { etc.), allg. Informationen zur } \\
\text { Organisation der Börse und } \\
\text { ihren Marktsegmenten }\end{array}$ \\
\hline NASDAQ & $\bullet$ & $\bullet$ & $\begin{array}{l}\text { Nationale und } \\
\text { wichtige } \\
\text { internationale Indizes }\end{array}$ & $\begin{array}{l}\text { Hitlisten, } \\
\text { Marktstatistiken } \\
\text { (wöchentl.), Prognosen, } \\
\text { Marktalarm }\end{array}$ & $\begin{array}{l}\text { Investor-Training, } \\
\text { Marktsimulationen }\end{array}$ \\
\hline NYSE & $\bullet$ & $\bullet$ & $\begin{array}{l}\text { Alle wichtigen } \\
\text { nationalen Indizes } \\
\text { (Dow Jones, AMEX, } \\
\text { S\&P 500, etc.) }\end{array}$ & $\begin{array}{l}\text { Umfangreiche } \\
\text { Marktstatistiken und } \\
\text {-analysen, Pressemit- } \\
\text { teilungen zu notierten } \\
\text { Unternehmen }\end{array}$ & $\begin{array}{l}\text { Pressemitteilungen, WWW- } \\
\text { Links, Einzelwerte auf } \\
\text { kostenpflichtiger CD, } \\
\text { Publikationen online } \\
\text { bestellbar, Umsatzvolumen } \\
\text { ab } 1888 \text { auf Tagesbasis }\end{array}$ \\
\hline Swiss Exchange & 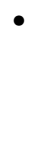 & & $\begin{array}{l}\text { Nationale Indizes } \\
\text { (SMI, SPI, SBI) }\end{array}$ & $\begin{array}{l}\text { Marktanalysen zu } \\
\text { inländ. und ausländ. } \\
\text { Aktien (monatl. } \\
\text { Bericht) }\end{array}$ & News, Presseinformationen \\
\hline Tokyo Stock & $\bullet$ & $\bullet$ & Nationale Indizes & Aktuelle Marktdaten & WWW-Links zu \\
\hline
\end{tabular}




\begin{tabular}{|c|c|c|c|c|c|}
\hline Exchange & & & (TOPIX, etc.) & $\begin{array}{l}\text { (Handelsvolumina, } \\
\text { Preise, etc.), } \\
\text { Marktstatistiken, } \\
\text { Jahresbericht, } \\
\text { Informationen zu } \\
\text { notierten Unternehmen }\end{array}$ & $\begin{array}{l}\text { Finanzinstitutionen und } \\
\text { internationalen Börsen }\end{array}$ \\
\hline $\begin{array}{l}\text { Baden- } \\
\text { Württembergische } \\
\text { Börse zu Stuttgart }\end{array}$ & $\bullet$ & $\bullet$ & $\begin{array}{l}\text { DAX, SAX und } \\
\text { internationale Indizes }\end{array}$ & Marktkommentar & $\begin{array}{l}\text { Umfangreiche WWW-Links } \\
\text { (z.B. zu Broker, Banken) } \\
\text { Presseberichte, } \\
\text { Szenarioanalyse } \\
\end{array}$ \\
\hline Bayerische Börse & $\bullet$ & $\bullet$ & DAX 30 & $\begin{array}{l}\text { WWW-Links zu } \\
\text { neueingeführten } \\
\text { Unternehmen }\end{array}$ & $\begin{array}{l}\text { Pressemeldungen, } \\
\text { Geschäftsbericht, WWW- } \\
\text { Links zu Informations- } \\
\text { diensten und } \\
\text { Finanzinstitutionen, } \\
\text { Börsenlexikon }\end{array}$ \\
\hline Berliner Börse & $\bullet$ & & & $\begin{array}{l}\text { Links und } \\
\text { Informationen zu } \\
\text { Emittenten }\end{array}$ & $\begin{array}{l}\text { Newsletter, Adressen und } \\
\text { Telefonnummern von } \\
\text { Finanzinstitutionen, WWW- } \\
\text { Links zu } \\
\text { Informationsdiensten im } \\
\text { Internet, Amtliches } \\
\text { Kursblatt }\end{array}$ \\
\hline $\begin{array}{l}\text { Bremer } \\
\text { Wertpapierbörse }\end{array}$ & - & - & $\begin{array}{l}\text { DAX, MDAX, } \\
\text { EuroStoxx, Dow } \\
\text { Jones }\end{array}$ & $\begin{array}{l}\text { Links und } \\
\text { Informationen zu } \\
\text { Emittenten }\end{array}$ & $\begin{array}{l}\text { WWW-Links zu } \\
\text { Börsenmitgliedern, } \\
\text { Devisenkurse, Amtliches } \\
\text { Kursblatt }\end{array}$ \\
\hline $\begin{array}{l}\text { Hanseatische } \\
\text { Wertpapierbörse } \\
\text { Hamburg }\end{array}$ & - & & & 2 & Seminare \\
\hline $\begin{array}{l}\text { Niedersächsische } \\
\text { Börse zu Hannover }\end{array}$ & - & & & & $\begin{array}{l}\text { Bücher, } \\
\text { Informationsmaterial über } \\
\text { Börsenhandel, WWW- } \\
\text { Links, Amtliches Kursblatt }\end{array}$ \\
\hline $\begin{array}{l}\text { Rheinisch- } \\
\text { Westfälische Börse } \\
\text { zu Düsseldorf }\end{array}$ & $\bullet$ & $\bullet$ & DAX 30 & & $\begin{array}{l}\text { Wichtige } \\
\text { Unternehmensmeldungen }\end{array}$ \\
\hline
\end{tabular}

${ }^{1}$ nur über kostenpflichtigen Service erhältlich ${ }^{2}$ Rubrik vorgesehen, jedoch zum Analysezeitpunkt keine Dokumente enthalten

Tabelle 3: Informationsspektrum

\subsection{Zeitnähe}

Die folgende Tabelle 4 verdeutlicht das Angebot an zeitnahen Informationen bzw. Echtzeitkursen im Internet. In der Regel werden Kursdaten einzelner Unternehmen mit einer zeitlichen Verzögerung von mindestens 15 Minuten angezeigt. Bei den jeweiligen Indizes ist eine zunehmende Verbreitung von Echtzeitdaten zu beobachten.

Im Hinblick auf die zeitliche Verzögerung der Daten ist die Tokyo Exchange besonders hervorzuheben, die Indizes und Kurse der einzelnen Unternehmen in Echtzeit anbietet. Die Amsterdam Exchange und die Bolsa de Madrid stellen Echtzeitkurse hingegen kostenpflichtig zur Verfügung. Madrid bietet diese sowie historische Kurse und Marktstatistiken jedoch nur über eine telefonische Hotline an. 


\begin{tabular}{|c|c|c|c|}
\hline \multirow[t]{2}{*}{ BÖRSE } & \multicolumn{2}{|c|}{ ZEITLICHE VERZÖGERUNG } & \multirow{2}{*}{ ANMERKUNGEN } \\
\hline & KURSE & INDEX $^{5}$ & \\
\hline Amsterdam Exchange & Echtzeit & Echtzeit & $\begin{array}{l}\text { Echtzeitkurse sind kostenpflichtig; } \\
\text { kostenlose Kurse sind } 20 \text { min verzögert. }\end{array}$ \\
\hline Bolsa de Madrid & mind. 15 min & mind. $15 \mathrm{~min}$ & $\begin{array}{l}\text { Echtzeitwerte über eine kostenpflichtige } \\
\text { Hotline abrufbar }\end{array}$ \\
\hline Borsa Italia & $15 \mathrm{~min}$ & $15 \mathrm{~min}$ & \\
\hline Bourse de Paris & keine Angabe & mind. $15 \mathrm{~min}$ & \\
\hline $\begin{array}{l}\text { Frankfurter } \\
\text { Wertpapierbörse }\end{array}$ & $15 \min$ & Echtzeit & \\
\hline $\begin{array}{l}\text { London Stock } \\
\text { Exchange }\end{array}$ & keine Kursdaten & keine Indizes & Kurse u.a. auf BBC-Website erhältlich. \\
\hline NASDAQ & $15 \mathrm{~min}$ & Echtzeit & \\
\hline NYSE & $20 \min$ & $\max .5 \min$ & \\
\hline Swiss Exchange & $30 \mathrm{~min}$ & Echtzeit & \\
\hline Tokyo Stock Exchange & Echtzeit & Echtzeit & \\
\hline $\begin{array}{l}\text { Baden- } \\
\text { Württembergische } \\
\text { Börse zu Stuttgart }\end{array}$ & $15 \min$ & $\begin{array}{l}\text { DAX und internationale Indizes in } \\
\text { Echtzeit; SAX vom Vortag und } \\
\text { historisch. }\end{array}$ & \\
\hline Bayerische Börse & mind. $15 \min$ & keine Indizes & \\
\hline Berliner Börse & mind. $15 \min$ & keine Indizes & \\
\hline $\begin{array}{l}\text { Bremer } \\
\text { Wertpapierbörse }\end{array}$ & mind. $15 \min$ & DAX mindestens 15 Minuten & \\
\hline $\begin{array}{l}\text { Hanseatische } \\
\text { Wertpapierbörse } \\
\text { Hamburg }\end{array}$ & 15 min bis 1,5 Std. & keine Indizes & \\
\hline $\begin{array}{l}\text { Niedersächsische } \\
\text { Börse zu Hannover }\end{array}$ & Vortag & keine Indizes & $\begin{array}{l}\text { Schlußkurse können börsentäglich ab } \\
18.00 \text { heruntergeladen werden. }\end{array}$ \\
\hline $\begin{array}{l}\text { Rheinisch- } \\
\text { Westfälische Börse zu } \\
\text { Düsseldorf }\end{array}$ & mind. 15 min & mind. 15 min & \\
\hline
\end{tabular}

Tabelle 4: Zeitverzögerung von Kursdaten und Indizes

\section{Abschließende Betrachtungen}

Zusammenfassend läßt sich eine Heterogenität zwischen den verschiedenen Börseninstitutionen sowohl bezüglich der Art der Informationen als auch deren Darstellung

\footnotetext{
${ }^{5}$ Es handelt sich jeweils um den ,eigenen“ Index der angesprochenen Börse.
} 
konstatieren. Insbesondere die Websites der Bourse de Paris, der Tokyo Stock Exchange, der FWB und der nordamerikanischen Börsen NASDAQ und NYSE zeichnen sich durch eine übersichtliche und ansprechende Darstellung ihrer Informationen unter Zuhilfenahme vielfältiger Charts, Tabellen und Kurzzusammenfassungen aus. Bei der Bourse de Paris profitiert der private Investor von einer Fülle an Informationen zu den notierten Unternehmen, die von verständlich formulierten Marktbeschreibungen über Branchenstatistiken und Bilanzdaten bis hin zu Pressedossiers reichen. Diese Informationen sind zur besseren Übersichtlichkeit für jedes Unternehmen jeweils auf einer Site zusammengefaßt.

Bei den Regionalbörsen bieten die Stuttgarter, die Münchner und die Berliner Börse umfangreiche und ansprechend aufbereitete Informationen und müssen den internationalen Vergleich nicht scheuen. Die restlichen deutschen Regionalbörsen beschränken sich hingegen auf ein Minimum an Informationen; neben Kursdaten bieten sie nur wenige Fakten zu Märkten, Unternehmen oder ergänzende Services für den Privatanleger an.

Vergleicht man die vorliegenden Ergebnisse mit den Resultaten einer Analyse, die im Dezember 1998 von den Autoren durchgeführt wurde, zeigen sich bei vielen Börsen Verbesserungen, die insbesondere das Layout und die Funktionalität betreffen. Eine grundsätzliche Umgestaltung des Angebotes hin zu einer verstärkten Orientierung am privaten Investor konnte jedoch nicht beobachtet werden. Eine Ausnahme bildet hier die FWB, die neben optischen Korrekturen ein deutlich erweitertes Spektrum an Informationen speziell für private Investoren bietet.

Nach [SeSt98] kann bei der Bereitstellung von Informationen zwischen uni- und bidirektionaler Kommunikation unterschieden werden. Als besondere Form bidirektionaler Kommunikation ist die kundenindividuelle Ansprache möglich. Gerade die bidirektionale Kommunikation wird jedoch kaum genutzt. Dies gilt sowohl für die untersuchten internationalen Börseninstitutionen als auch für die Regionalbörsen, obwohl sich insbesondere diese oftmals als „Börse für den privaten Anleger“ präsentieren.

Primäre Adressaten des Internetauftritts der Börseninstitutionen sind immer noch deren direkte Kunden, konkret die Broker und Banken, die in der Intermediationskette zwischen Privatkunde und Börse agieren, sowie die großen, institutionellen Investoren. Weiterhin steigende Anteile privater Investoren am gesamten Wertpapierhandel und die Tatsache, daß auch dem privaten Investor zunehmend die Bedeutung der Auswahl des Börsenplatzes für die Geschwindigkeit und Preisqualität seiner Transaktionen bewußt wird, erfordern jedoch auch die Bereitstellung neuartiger, speziell auf die Bedürfnisse anspruchsvoller Kunden 
zugeschnittene Informationsangebote, um im Wettbewerb auf dem „Markt für Märkte“ gerade auch vor dem Hintergrund der Konkurrenz durch private elektronische Handelsplattformen - bestehen zu können.

\section{Literatur}

[Birk96] Birkelbach, J.: Angriff auf das Monopol, in: SPIEGEL Special, Nr. 5 (1996), S. 98.

[DAI99] Deutsches Aktieninstitut: DAI-Factbook 1999 - Statistiken, Analysen und Grafiken zu Aktionären, Aktiengesellschaften und Börsen. Deutsches Aktieninstitut 1999.

[DB2000] Deutsche Börse AG: Gesamtumsätze in Aktien für das Jahr 1999. Einzelanfrage historischer Daten. Frankfurt, Januar 2000.

[Go2000] Gomber, P.: Elektronische Handelssysteme - Innovative Konzepte und Technologien im Wertpapierhandel. Heidelberg 2000.

[GrBi98] Grob, H. L.; Bieletzke, S.: Erfolgsfaktoren für das System Internet, in: Hippner, H. et al. (Hrsg.): Computer Based Marketing. Braunschweig/Wiesbaden 1998, S. 109-116.

[Metz94] Metzger, J.: US-Börsen, in: Beiträge zur Theorie der Finanzmärkte, Institut für Kapitalmarktforschung, J. W. Goethe Universität, Frankfurt 1994.

[SeSt98] Seitz, J.; Stickel, E.: WWW als neuer Vertriebsweg für Finanzdienstleister, in: Hippner, H. et al. (Hrsg.): Computer Based Marketing. Braunschweig/Wiesbaden 1998, S. 405-412.

[voRo94] von Rosen, R.: Private Handelssysteme - eine Herausforderung für die Börsen. In: Zeitschrift für das gesamte Kreditwesen 24 (1994), S. 1213-1217.

[WeGo99] Weinhardt, Ch.; Gomber, P.; Holtmann, C.; Groffmann, H. D.: Online Brokerage - Phasenintegration als strategische Chance. Erscheint in: LocarekJunge, H. (Hrsg.): Banken im Wandel: Direkt Banking und Direktbanken, Berlin, 2000. 